\title{
DIFFUSIVE SOLUTIONS OF THE COMPETITIVE LOTKA-VOLTERRA SYSTEM
}

\author{
LI-CHANG HUNG
}

Abstract. In the present work we show by means of explicit construction that three new types of solutions exist for the one dimensional competitive Lotka-Volterra reaction-diffusion system. The new solutions constructed are (i) space-time separated solutions, (ii) unbounded solutions, and (iii) solutions of Gaussian type, with the constructions being based largely on the standard methods for constructing solutions to the one-dimensional heat equation. From these exact solutions a new and interesting phenomena is found, namely diffusion-induced long-term coexistence of three species. In addition, the approach to constructing explicit solutions presented here can readily be applied to other reaction-diffusion systems.

Mathematics subject classification (2010): 35K55, 35K57, 35K60.

Keywords and phrases: time-periodic unbounded solution, solution of Gaussian type, Lotka-Volterra, explicit solution, heat equation, reaction-diffusion system.

\section{REFERENCES}

[1] M. J. ABlowitz AND A. Zeppetella, Explicit solutions of Fisher's equation for a special wave speed, Bull. Math. Biol., 41 (1979), pp. 835-840.

[2] D. G. Aronson AND H. F. Weinberger, Nonlinear diffusion in population genetics, combustion, and nerve pulse propagation, in Partial differential equations and related topics (Program, Tulane Univ., New Orleans, La., 1974), Springer, Berlin, 1975, pp. 5-49. Lecture Notes in Math., Vol. 446.

[3] C.-C. Chen, L.-C. Hung, M. Mimura, M. Tohma, And D. Ueyama, Semi-exact equilibrium solutions for three-species competition-diffusion systems, submitted.

[4] C.-C. Chen, L.-C. Hung, M. Mimura, And D. Ueyama, Exact traveling wave solutions of three species competition-diffusion systems, to appear in Discrete and Continuous Dynamical System-B.

[5] R. M. CherNiGa AND V. A. DutKA, A diffusive Lotka-Volterra system: Lie symmetries, exact and numerical solutions, Ukraïn. Mat. Zh., 56 (2004), pp. 1395-1404.

[6] R. Cherniha AND V. DAVydovych, Conditional symmetries and exact solutions of the diffusive Lotka-Volterra system, Mathematical and Computer Modelling, 54 (2011), pp. 1238 - 1251.

[7] R. CheRniHA AND V. DAVYDOVYCH, Lie and conditional symmetries of the three-component diffusive Lotka-Volterra system, Journal of Physics A: Mathematical and Theoretical, 46 (2013), p. 185204.

[8] N. FEI AND J. CARR, Existence of travelling waves with their minimal speed for a diffusing LotkaVolterra system, Nonlinear Anal. Real World Appl., 4 (2003), pp. 503-524.

[9] P. FIFE AND J. MCLEOD, The approach of solutions of nonlinear diffusion equations to travelling front solutions, Archive for Rational Mechanics and Analysis, 65 (1977), pp. 335-361.

[10] P. C. FIFE, Mathematical aspects of reacting and diffusing systems, vol. 28 of Lecture Notes in Biomathematics, Springer-Verlag, Berlin, 1979.

[11] R. A. FISHER, The wave of advance of an advantageous gene, Ann. Eugen, 7 (1936), pp. 355-369.

[12] X. Hou AND A. W. LeUnG, Traveling wave solutions for a competitive reaction-diffusion system and their asymptotics, Nonlinear Anal. Real World Appl., 9 (2008), pp. 2196-2213.

[13] F. John, Partial differential equations, vol. 1 of Applied Mathematical Sciences, Springer-Verlag, New York, fourth ed., 1982.

[14] Y. KAN-ON, Parameter dependence of propagation speed of travelling waves for competition-diffusion equations, SIAM J. Math. Anal., 26 (1995), pp. 340-363. 
[15] - Existence of standing waves for competition-diffusion equations, Japan J. Indust. Appl. Math., 13 (1996), pp. 117-133.

[16] - Fisher wave fronts for the Lotka-Volterra competition model with diffusion, Nonlinear Anal., 28 (1997), pp. 145-164.

[17] J. I. KANEL, On the wave front solution of a competition-diffusion system in population dynamics, Nonlinear Anal., 65 (2006), pp. 301-320.

[18] J. I. KANEL AND L. ZHOU, Existence of wave front solutions and estimates of wave speed for a competition-diffusion system, Nonlinear Anal., 27 (1996), pp. 579-587.

[19] A. Kolmogoroff, I. Petrovsky, And N. Piscounoff, Study of the diffusion equation with growth of the quantity of matter and its application to a biological problem.(French) Moscow Univ, Bull. Math, 1 (1937), pp. 1-25.

[20] A. W. Leung, X. Hou, AND W. Feng, Traveling wave solutions for Lotka-Volterra system revisited, Discrete Contin. Dyn. Syst. Ser. B, 15 (2011), pp. 171-196.

[21] A. W. LEUNG, X. Hou, AND Y. LI, Exclusive traveling waves for competitive reaction-diffusion systems and their stabilities, J. Math. Anal. Appl., 338 (2008), pp. 902-924.

[22] S. MARTínEZ, The effect of diffusion for the multispecies Lotka-Volterra competition model, Nonlinear Anal. Real World Appl., 4 (2003), pp. 409-436.

[23] J. D. Murray, Mathematical biology, vol. 19 of Biomathematics, Springer-Verlag, Berlin, second ed., 1993.

[24] M. Rodrigo and M. Mimura, Exact solutions of a competition-diffusion system, Hiroshima Math. J., 30 (2000), pp. 257-270.

[25] M. Rodrigo AND M. Mimura, Exact solutions of reaction-diffusion systems and nonlinear wave equations, Japan J. Indust. Appl. Math., 18 (2001), pp. 657-696.

[26] M. TANG AND P. FIFE, Propagating fronts for competing species equations with diffusion, Archive for Rational Mechanics and Analysis, 73 (1980), pp. 69-77.

[27] J. H. VAN VUUREN, The existence of travelling plane waves in a general class of competition-diffusion systems, IMA J. Appl. Math., 55 (1995), pp. 135-148.

[28] A. I. Volpert, V. A. Volpert, AND V. A. Volpert, Traveling wave solutions of parabolic systems, vol. 140 of Translations of Mathematical Monographs, American Mathematical Society, Providence, RI, 1994. Translated from the Russian manuscript by James F. Heyda.

[29] J. Y. Wakano, M. A. Nowak, And C. Hauert, Spatial dynamics of ecological public goods, Proceedings of the National Academy of Sciences, 106 (2009), pp. 7910-7914.

[30] I. YAnOvsKy, Partial Differential Equations: Graduate Level Problems and Solutions. http://www.math.ucla.edu/〜yanovsky/handbooks/, 2005. 